\title{
Catamenial pneumothorax - are there benefits of cooperation between the surgeon and the gynaecologist?
}

\author{
Cezary Miedziarek ${ }^{1}$, Mariusz Kasprzyk ${ }^{2}$ \\ 1Student Research Group of Thoracic Surgery, Faculty of Medicine, Poznan University of Medical Sciences, Poznan, Poland \\ ${ }^{2}$ Department of Thoracic Surgery, Poznan University of Medical Sciences, Poznan, Poland
}

\begin{abstract}
Catamenial pneumothorax (CP) is an uncommon disease related to thoracic endometriosis or diaphragmatic perforation. It is defined as an occurrence of spontaneous pneumothorax around the time of menstruation in the population of women of reproductive age with no other lung diseases. Catamenial pneumothorax is often misdiagnosed and classified as a spontaneous pneumothorax, and no in-depth gynaecological diagnostic is performed. Here we present 3 cases of female patients admitted to the Department of Thoracic Surgery with the aim of surgical treatment of recurrent CP. In all 3 cases, a temporal association between pneumothorax and menstruation was identified. In 2 presented cases the patients were diagnosed with endometriosis and other gynaecological diseases. The previous incidents of CP were treated conservatively, with suction drainage of the pleural cavity or with thoracocentesis, but the treatment did not prevent relapses. In the Department of Thoracic Surgery, each patient was operated on with the use of video-assisted thoracoscopic surgery, which is considered to be a good therapeutic option. In the case of 2 patients the surgery eliminated the relapses, 1 patient required radical lung decortication. The literature on the subject indicates the potential benefits of hormonal treatment of CP. It is suggested that using such pharmacological treatment may reduce the risk of relapse after surgery.
\end{abstract}

Key words: pneumothorax, catamenial pneumothorax, endometriosis, surgery.

\section{Introduction}

Catamenial pneumothorax (CP) is defined as the appearance of air in the pleural cavity in women of reproductive age with no other lung diseases, occurring 72 hours before or after menstruation. The literature indicates also other time criteria - up to 7 days before and after monthly bleeding [1]. The condition concerns 3-6\% of menstruating women with spontaneous pneumothorax and is more often diagnosed in women referred to the surgery ward (25-30\%) [2]. Catamenial pneumothorax may be associated with thoracic endometriosis or diaphragmatic perforation [1, 2]. The condition is often misdiagnosed and classified as spontaneous pneumothorax due to lack of analysis of patients' menstrual history and failure to perform a routine intraoperative diaphragm inspection [3]. Proper CP diagnosis may be crucial to the patient even after surgical treatment of pneumothorax because hormonal therapy may contribute to the avoidance of recurrence of CP incidents [4]. Here we present 3 cases of recurrent CP treated surgically in the Thoracic Surgery Ward and briefly summarize the current state of knowledge on the subject.

\section{Cases presentation}

\section{Case 1}

A 36-year-old female patient was referred to the Thoracic Surgery Ward with the aim of surgical treatment of the third episode of spontaneous right-sided pneumothorax. Her complaints included chest pain and dyspnoea. The first episode, treated with suction drainage of the pleural cavity, occurred 4 months earlier and the second one, treated with thoracocentesis, a month earlier. The patient indicated the time association between the pneumothorax incidents and menstruation. Her past medical history included several laparoscopies due to endometriosis and ovarian endometrial cysts, infertility treatment, and caesarean sections, all resulting in the presence of numerous adhesions in the abdominal cavity. Chest $X$-ray showed a right pneumothorax with a $5.2-\mathrm{cm}$ gap at the top of the lung. At the Thoracic Surgery Ward, the patient was treated with right-sided video-assisted thoracoscopic surgery (VATS). During the surgery, a fibrous lesion at the top of the lung and a cystic lesion on the diaphragm were observed. There was no emphysema or air leak. Pleurectomy was performed 


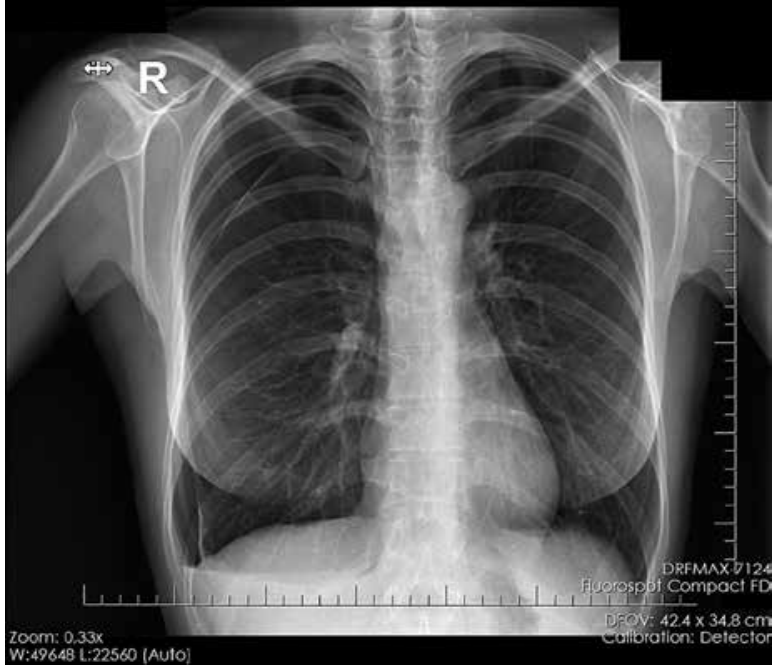

Fig. 1. Patient 1 - preoperative chest $\mathrm{X}$-ray

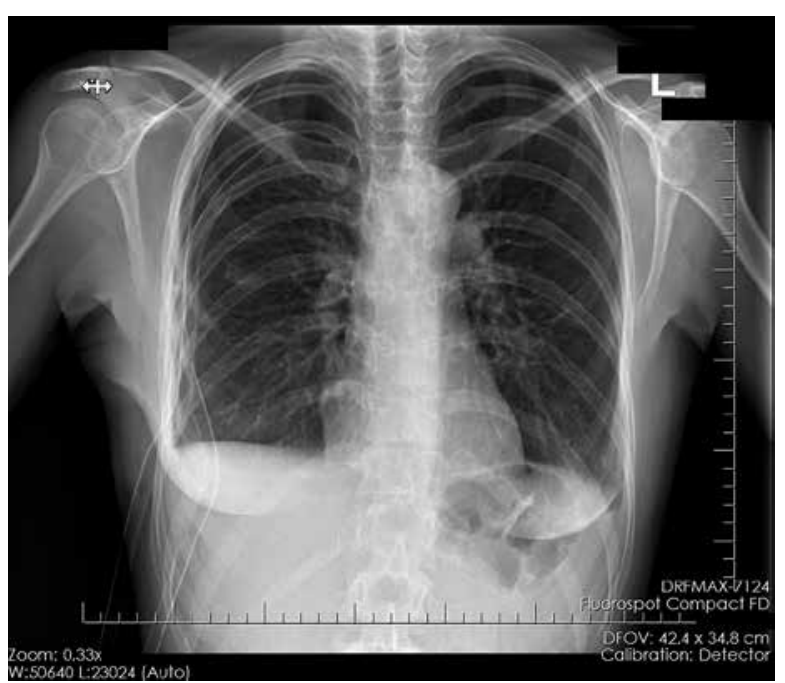

Fig. 2. Patient 1 - postoperative chest $\mathrm{X}$-ray (day 2 after surgery)

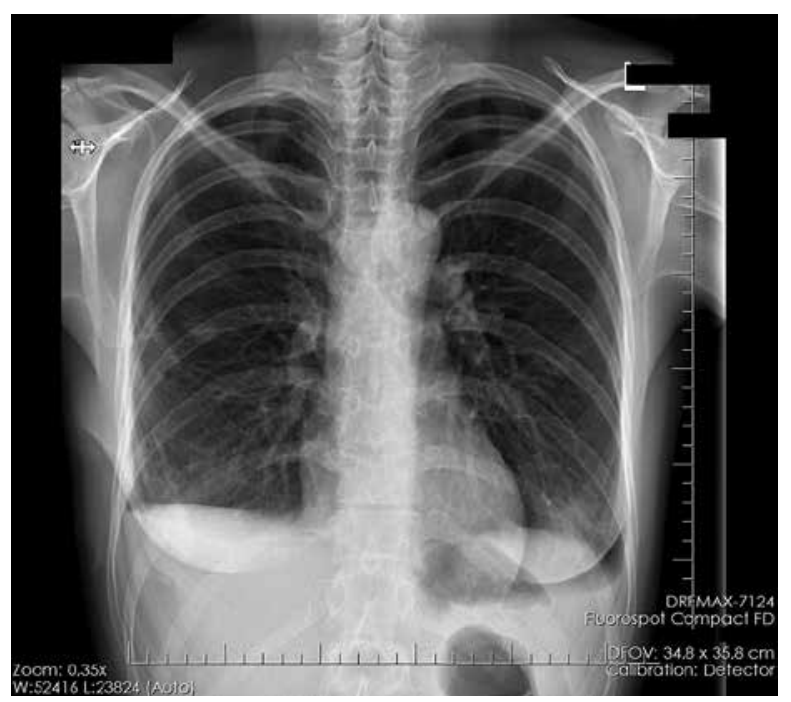

Fig. 3. Patient 1 - postoperative chest X-ray (day 3 after surgery) and the sections of the pleura were sent to histopathological examination, which later revealed a lymphocytic inflammatory infiltration. Mechanical and talc pleurodesis was applied. The patient was discharged from the hospital in good condition (Fig. 1-3).

\section{Cose 2}

A 46-year-old female patient was urgently admitted to the Thoracic Surgery Ward with the aim of surgical treatment of a fourth episode of spontaneous right-sided pneumothorax. The recent incidents took place 3, 5, and 7 months before the admission and were treated with suction drainage of the pleural cavity. The patient complained about dyspnoea. After analysis, the temporal association between pneumothorax incidents and menstruation was found. The patient was unsuccessfully treated with hormonal therapy for endometriosis and abnormal uterine bleeding. Chest X-ray showed a right pneumothorax with a size of $2.0 \mathrm{~cm}$. Right-sided VATS was performed, during which a pleural adhesion was visible at the top of the lung. The lesion was resected, and histopathological examination revealed an unspecific inflammation with mesothelial hyperplasia. A wedge resection from the 6th segment of the right lung, as well as pleurectomy, and mechanical and talc pleurodesis were performed. Histopathological examination of the lung parenchyma revealed atelectasis and fresh bleeding to the pulmonary alveoli. There were no postoperative complications.

\section{Case 3}

A 40-year-old patient was admitted to the Thoracic Surgery Ward for surgical treatment of recurrent spontaneous right-sided pneumothorax. The patient was conservatively treated 12 times before admission, each month during menstruation. Each time her symptoms included chest pain and dyspnoea, especially during physical activity. Endometriosis or any other diseases were not diagnosed in the patient. Chest X-ray showed a right pneumothorax with a size of $3.6 \mathrm{~cm}$. Video-assisted thoracoscopic surgery was performed and revealed congested lung tissue with abnormal density. Lung biopsy and pleurectomy were made. Histopathological examination revealed atelectasis, bleeding to the pulmonary alveoli, and lymphocytic inflammatory infiltration of the pleura. The patient presented to the Thoracic Surgery Ward a year later with similar symptoms as previously; chest X-ray showed no pathological lesions. She was admitted to the ward with the aim of performing total lung decortication, which was performed. The patient was not hospitalized since the second operation (9 months of observation). 


\section{Discussion}

In most cases (93\%), CP occurs in the right lung, which can be explained by the retrograde menstruation (migration) theory of CP pathogenesis, which combines the fact of the clockwise directionality of peritoneal flow with retrograde menstruation. There are also other theories explaining CP pathogenesis. The prostaglandin theory assumes that a high level of prostaglandin F2 during menstruation may induce vasoconstriction and bronchospasm resulting in damage to the pulmonary lesions. Another theory implies that pleura, similarly to other tissues of coelomic origin, may differentiate into endometrial cells when exposed to a high level of oestrogens. The diaphragmatic theory of air passage and the theory of endometrial transplantation through lymphatic or vascular microembolization are also considered [5]. Pelvic endometriosis is a comorbid disease in only $55 \%$ of patients, which explains why the diagnosis of CP is still probable in Case 3 [6].

The diagnosis of CP is usually made based on patients' clinical status (symptoms of pneumothorax - shortness of breath, chest pain, shoulder pain) correlated, as in the presented cases, with the occurrence of symptoms during or around the time of menstruation [5]. It should be noted that CP is not the only possible manifestation of thoracic endometriosis. Other signs and symptoms include catamenial haemothorax, catamenial haemoptysis, pulmonary nodules, chest and shoulder pain, and radiologic findings [7].

Video-assisted thoracoscopic surgery is a minimally invasive surgical procedure, currently considered as more beneficial for the patients than open thoracotomy [8]. Video-assisted thoracoscopic surgery can be used both for diagnosis (e.g. thoracic lesion biopsy) and treatment of thoracic diseases. The indications to the surgical treatment with the use of VATS are predominantly related to pulmonary, pleural, oesophageal, and chest wall diseases [9]. Video-assisted thoracoscopic surgery is also considered to be a good diagnostic and therapeutic method in CP treatment. The most frequently performed surgical interventions specifically in CP management are pleurodesis, diaphragmatic interventions, diaphragm suture, lung resection with diaphragmatic placation, diaphragm resection, and apical wedge lung resection. The rarest types of surgical interventions include non-defined wedge resection and middle lobe resection. The endometrial implants are found intraoperatively in $59.3 \%$ of patients, and the histopathology confirms thoracic endometriosis in $52.3 \%$ of patients with CP. $25 \%$ of patients suffer from recurrence of the condition, which shows the necessity of patient follow-up [10].

It is believed that hormonal therapy should be used in the preoperative and postoperative treatment of CP. Gonadotrophin-releasing hormone $(\mathrm{GnRH})$ analogue therapy, which is used most frequently, applied after the surgery reduces the risk of recurrence. There are also studies suggesting that such therapy should be used only in selected cases. Other types of hormonal treatment, less widely used in CP treatment, include hormone contraceptives, GnRH agonist plus progesterone, progesterone, and danazol $[11,12]$. There are some reports proving the effectiveness of hysterectomy with bilateral salpingo-oophorectomy as a definitive treatment of CP [13]. British Thoracic Society pleural disease guidelines recommend a combination of surgical intervention and hormonal manipulation, emphasizing the fact that surgical treatment combined with GnRH analogues prevent the recurrence of $\mathrm{CP}$ in a follow-up period of up to 4 years. Amenorrhoea as a side effect of the therapy must be taken into consideration. As a comparison, surgical treatment implemented alone results in recurrence rates of up to $30 \%$ [14].

\section{Conclusions}

All theories of CP pathogenesis assume the role of endometrial tissue or the coexistence of endometriosis as a key factor in the development of the disease. It proves the reasonableness of gynaecological consultation of patients with CP with the objective of actively seeking endometriosis, as well as implementing hormonal treatment along with the surgical treatment. It is to be noted that due to the rarity of the disease and the common overlooking of the gynaecological cause of $\mathrm{CP}$ there are no randomized trials concerning the effectiveness of hormonal treatment, and further studies on the subject are needed. It is potentially beneficial to the patients by reducing the number of invasive procedures used in CP treatment.

\section{Disclosure}

The authors report no conflict of interest.

\section{References}

1. Marjański T, Sowa K, Czapla A, et al. Catamenial pneumothorax - a review of the literature. Pol J Thorac Cardiovasc Surg 2016; 13: 117-121.

2. Visouli AN, Darwiche K, Mpakas A, et al. Catamenial pneumothorax: a rare entity? Report of 5 cases and review of the literature. J Thorac Dis 2012; Suppl 1: 17-31.

3. Shrestha B, Shrestha S, Peters $P$, et al. catamenial pneumothorax, a commonly misdiagnosed thoracic condition: multicentre experience and audit of a small case series with review of the literature. Heart Lung Circ 2019; 28: 850-857.

4. Celik S, Erşen E. Catamenial pneumothorax. In: Amer K, ed. Pneumothorax. London: Intechopen; 2009: 1-14.

5. Narula N, Ngu S, Avula A, et al. Left-sided catamenial pneumothorax: a rare clinical entity. Cureus 2018; 10: e2567.

6. Gil Y, Tulandi T. Diagnosis and treatment of catamenial pneumothorax: a systematic review. J Minim Invasive Gynecol 2020; 27: 48-53.

7. Nezhat C, Lindheim SR, Backhus L, et al. thoracic endometriosis syndrome: a review of diagnosis and management. JSLS 2019; 23: e2019.00029. 
8. Al-Ameri M, Bergman P, Franco-Cereceda A, et al. Video-assisted thoracoscopic versus open thoracotomy lobectomy: a Swedish nationwide cohort study. J Thorac Dis. 2018; 10: 3499-3506.

9. Mehrotra M, D'Cruz JR, Arthur ME. Video-assisted thoracoscopy. StatPearls Publishing. https://www.ncbi.nlm.nih.gov/books/NBK532952/. Accessed August 4, 2021.

10. Bricelj K, Srpčič $M$, Ražem A, et al. Catamenial pneumothorax since introduction of video-assisted thoracoscopic surgery. Wiener Klin Wochenschrift 2017; 129: 717-726.

11. Subotic D, Mikovic Z, Atanasijadis N, et al. Hormonal therapy after the operation for catamenial pneumothorax - is it always necessary? J Cardiothorac Surg 2016; 11: 1-6.

12. Iyer S, Ehrig JC, Sosa M, et al. Operative and hormonal management of recurrent catamenial pneumothorax: a case report. Southwest Respir Crit Care Chronicles 2018; 6: 22-25.

13. MacDuff A, Arnold A, Harvey J. Management of spontaneous pneumothorax: British Thoracic Society pleural disease guideline 2010. Thorax 2010; 65: 18-31. 